\title{
HÁBITOS LECTORES Y GÉNEROS DEL DISCURSO EN FILOSOFÍA Y EN ECONOMÍA Y NEGOCIOS: DEL DISCURSO ACADÉMICO AL DISCURSO PRO- FESIONAL ${ }^{1}$
}

\author{
READING HABITS AND DISCOURSE GENRES IN PHILOSOPHY \\ AND IN ECONOMICS AND BUSINESS: FROM ACADEMIC TO \\ PROFESSIONAL DISCOURSE
}

\section{GIOVANNI PARODI}

Pontificia Universidad Católica de Valparaíso.

giovanni.parodi@pucv.cl

\section{GINA BURDILES}

Universidad Católica de la Santísima Concepción.

gburdiles@ucsc.cl

\section{TOMÁS MORENO-DE LEÓN}

Pontificia Universidad Católica de Valparaíso.

tomas.moreno.d@mail.pucv.cl

\section{CRISTÓBAL JULIO}

Pontificia Universidad Católica de Valparaíso.

cristobal.julio@pucv.cl

\section{RESUMEN}

Probablemente la lectura es uno de los medios fundamentales de acceso y construcción de conocimientos especializados, tanto en el mundo académico como en el profesional. Entonces, comprender el discurso escrito a través de sus diversos géneros disciplinares constituye un requisito primordial en el mundo globalizado contemporáneo, tanto en sus formatos en papel como en digital. En este contexto, hoy en día existe escasa investigación que explore, describa y contraste los hábitos lectores de estudiantes universitarios y de profesionales en ámbitos disciplinares diversos. Intentando aportar a llenar esta brecha, la presente investigación busca conocer y describir los hábitos de lectura de un grupo de 580 estudiantes universitarios y de un grupo de 46 profesionales, tomando en cuenta sus preferencias de lectura en papel o digital y los géneros del discurso en dos áreas disciplinares: filosofía y economía y negocios. Los principales resultados indican que los sujetos de

\footnotetext{
${ }^{1}$ Investigación financiada por el Proyecto FONDECYT 1170623
} 
ambos grupos dan muestras de tener conciencia y conocimiento de los mecanismos discursivos de acceso al conocimiento especializado y declaran emplear una amplia gama de géneros. Al mismo tiempo, se observa una relativa atomización y especialización en pocos géneros profesionales específicos en ambos dominios disciplinares, en contraste con la gran heterogeneidad identificada en el discurso académico. En términos generales, el formato papel es el preferido para tareas de estudio y aprendizaje, tanto por estudiantes como por profesionales, aunque los estudiantes y profesionales de economía y negocios declaran también preferir géneros digitales.

Palabras clave: Hábitos lectores, discurso académico, discurso profesional, géneros del discurso, discurso disciplinar, géneros digitales, géneros en papel.

\section{ABSTRACT}

Probably, reading is one of the fundamental means of accessing and constructing specialized knowledge, both in academic and professional environments. Then, comprehending written discourse through its diverse disciplinary genres is an essential requirement in the contemporary globalized world, both in paper and digital media. In this context, nowadays, there is scarce research that explores, describes and contrasts the reading habits of university students and professionals in diverse disciplinary fields. Attempting to fill this gap, this study describes the reading habits of a group of 580 university students and a group of 46 professionals in terms of reading on paper and digital, and the discourse genres in two disciplinary areas: philosophy and economics and business. The main results indicate that the subjects of both groups are awareness of the discourse mechanisms of accessing specialized knowledge and declare to employ a wide variety of genres. At the same time, a relative atomization and specialization is observed in a few specific professional genres in both disciplinary domains, in contrast to the great heterogeneity identified in academic discourse. In general terms, the paper medium is preferred for study and learning tasks, both by students and professionals, although students and professionals in business and economics declare that they also prefer digital genres.

Keywords: Reading habits, academic discourse, professional discourse, discourse genres, disciplinary discourse, digital genres, paper genres.

Recibido: 25.05.2018. Aceptado: 20.10.2018.

\section{INTRODUCCIÓN}

L a nueva agenda 2015-2030 de las Naciones Unidas para el desarrollo sostenible establece, como parte de sus objetivos, que una educación de calidad es la base para mejorar la vida de las personas (Naciones Unidas, 2016). Específicamente, dentro de las metas del Objetivo 4 se declara que, para el año 2030, en el área de lenguaje, se busca garantizar que todos los jóvenes y, al menos una proporción 
sustancial de los adultos, tengan competencias de lectura y escritura. Desde este marco, en los últimos tiempos los resultados obtenidos a partir de diversos instrumentos revelan un escenario de desarrollos un tanto dispar para contribuir a los objetivos de las NU. Aunque recientemente pudiera existir un cierto énfasis en las investigaciones con foco en escritura $y$, comparativamente, se revele una falta de atención a la lectura (Arnoux, 2016; Tapia, Ávila, Navarro y Bazerman, 2016; Navarro, Ávila, Tapia-Ladino, Cristovão, Moritz, Narváez y Bazerman, 2016), es evidente que ambas habilidades requieren mayor atención inminente.

Si bien en la actualidad se puede sostener que el acceso a la información y, por ende, a la eventual construcción de conocimientos disciplinares se ha democratizado en términos de la disponibilidad que ofrece la internet, aún es fragmentaria la investigación acerca de los efectos de una transición de lectura en papel a una en diversos soportes electrónicos. Por el momento, se dispone de escasa información científica robusta que reporte qué se lee en digital y qué se lee en papel y para qué propósitos se emplea una y otra información. En este contexto, desde una perspectiva de formación continua, los hábitos de lectura constituyen parte esencial de la formación académica y profesional y se instalan como sustento importante de la vida laboral en todos los ámbitos del conocimiento (Parodi, 2014; Parodi, Moreno-de León, Julio y Burdiles, en prensa). Por tanto, como un modo de aportar a llenar este vacío, en esta investigación se busca conocer y describir los hábitos de lectura de un grupo de estudiantes universitarios y de un grupo de profesionales en cuanto a lectura en papel y en digital y a empleo de géneros del discurso, guiados por precisos propósitos académicos, en dos áreas disciplinares: filosofía y economía y negocios.

De modo más específico, tres preguntas de investigación guían el presente estudio:

1. ¿Existen diferentes hábitos de lectura en términos del tipo de formato (papel/ digital) entre lectores de disciplinas diversas?

2. ¿Existen diferencias entre la lectura en papel y en digital entre estudiantes y profesionales de filosofía y de economía y negocios?

3. ¿Qué géneros discursivos declaran leer en papel y en digital estudiantes y profesionales de filosofía y de economía y negocios.

Para responder a ellas, en la primera parte de este artículo, con el fin de encuadrar teóricamente la investigación, se entrega una sucinta pero actualizada revisión de la literatura respecto de discurso académico y discurso profesional escrito de la filosofía y de la economía y los negocios. Seguidamente, nos centramos en los aspectos metodológicos y damos cuenta de las muestras de lectores universitarios y profesionales, los instrumentos empleados para recoger los datos y los procedi- 
mientos implementados en cada caso. A continuación, describimos los resultados y discutimos los principales hallazgos y los relacionamos con la literatura especializada. Por último, cerramos con una síntesis de conclusiones, proyecciones y limitaciones.

\section{MARCO DE REFERENCIA}

\subsection{Discurso académico y discurso profesional}

La investigación sobre discurso especializado destaca por su atención en el uso del lenguaje en contextos académicos, enfocándose en los factores que, según Bhatia (2002), definen a este tipo de discurso, a saber, sus rasgos lexicogramaticales, sus variaciones disciplinares, los constructos genéricos que les son propios y los procedimientos pedagógicos para su enseñanza/aprendizaje. Aunque con mayor énfasis en la escritura, los estudios sobre discurso académico y alfabetización disciplinar en español son numerosos (e.g., Cubo de Severino, 2005; Parodi, 2010, 2015; Moyano, 2013; Montolío, 2014; Parodi y Burdiles 2015; Arnoux, 2016; Tapia et al., 2016; Navarro et al., 2016). En términos generales, el discurso académico se identifica con las variedades de lengua que se emplean para interactuar en contextos educativos a fin de acceder al conocimiento especializado propio del quehacer de la academia (Berkenkotter, Huckin y Ackerman, 1991; Swales, 1990, 2004; Flowerdew, 2002; Bhatia, 2002; Hyland, 2000, 2011; Silver, 2006).

Desde hace más de dos décadas, también se ha puesto de manifiesto la necesidad de profundizar en el conocimiento de los discursos profesionales (e.g., Bazerman y Paradis, 1991; Hyland, 2000; Becher y Trowler, 2001; Parodi, 2009, 2015; Koester, 2010; Cassany y López, 2010; Montolío, 2011; Navarro, 2012, 2015; Bolívar y Parodi, 2015). No obstante ello, Cassany y López (2010: 349) afirmaban que "Disponemos de pocos estudios empíricos que describan las prácticas letradas laborales y mucho menos que las comparen con las realizadas en el seno de una institución académica”. A pesar de que han transcurrido algunos años, esta aseveración mantiene su vigencia.

En términos definicionales, el discurso profesional se presenta como un complejo objeto de estudio, lo que dificulta definirlo conceptualmente y determinar con precisión sus características distintivas (Koester, 2010; Breeze, 2015). De hecho, no es tarea simple establecer con nitidez cuáles son los límites entre el discurso académico y el profesional, puesto que se trata de prácticas comunicativas dinámicas, desarrolladas en contextos sociales multidimensionales y que, en algunos casos, se sobrecruzan (Flowerdew, 2002; Bolívar y Parodi, 2015). De este modo, en busca de delimitar sus fronteras, nos situaremos en el marco propuesto por Bo- 
lívar y Parodi (2015), quienes plantean que las principales distinciones están dadas por: el contexto de producción, el contexto de circulación, los participantes y sus interacciones, los propósitos comunicativos y la dimensión lingüístico/discursiva.

En esta línea, Bolívar y Parodi (2015) subrayan que, en su origen, el estudio del discurso profesional estuvo principalmente asociado al inglés y con especial atención, en un principio, a las interacciones orales; en parte, ello se debió a la influencia del análisis conversacional desde los enfoques de la investigación socioconstructivista. Los autores destacan que, a partir de la década del noventa, se comienzan a hacer visibles investigaciones sobre discurso profesional (Boden, 1994; Linell, 1998) y que, desde entonces, han seguido aumentando de manera constante, tanto desde perspectivas lingüísticas (Connor y Upton, 2004), como desde aproximaciones sociales y críticas (Sarangi, 2004). Junto a ello, también han surgido propuestas a favor de estudiar el discurso profesional de manera multidisciplinaria, incorporando al análisis lingüístico los aportes de la sociología, el constructivismo social, la psicología cognitiva, la teoría organizacional, entre otras (Sales, 2006; Gunnarsson, 2009; Orna-Montesinos, 2012; Kong, 2014).

En la literatura disponible, destacan -entre otros- los trabajos que abordan el discurso profesional desde un enfoque de género, dando cuenta de los propósitos comunicativos y los rasgos lingüístico-textuales que coocurren sistemáticamente en algunos textos propios de ciertos ámbitos profesionales (Bhatia, 1993; Trosborg, 2000; Gil-Salom y Soler-Monreal, 2014; López-Ferrero y Bach, 2016). Asimismo, sobresalen investigaciones que abordan el discurso profesional en disciplinas particulares, como es el caso del discurso del diseño de información (Spinuzzi, 2003) y su variante diseño de información con finalidad educativa (Rouet, Lowe y Schnot, 2010), de la ingeniería (Sales, 2006), de la terapia sicoanalítica (Arnoux, Di Stefano y Pereira, 2010), de la medicina (Burdiles, 2012, 2016), del discurso jurídico (Montolío, 2011; Bhatia, Garzone, Salvi, Tessuto y Williams, 2014) y del discurso organizacional (Pieterse, 2014).

Ahora bien, desde el foco específico del presente estudio, se observa que tanto el discurso académico como el discurso profesional de la filosofía han sido escasamente estudiados desde consideraciones lingüísticas. Sí se ha abordado el análisis del discurso de la filosofía en su articulación entre el discurso de la modernidad y el discurso postmoderno. Al respecto, Neubert y Reich (2002: 22) consideran al discurso filosófico postmoderno ".... as inquiry into the validity claims of specific bodies of knowledge and historically changeable forms of truth". En este sentido, para Neubert y Reich (2002), la reflexión filosófica se dispone como un discurso eminentemente argumentativo y está intrínsecamente anclado en cada cultura, en oposición a la objetividad y universalidad. Según Reich (2000), en la perspectiva de Foucault (1972), el discurso filosófico postmoderno establece que la verdad y el conocimiento son plurales, contextuales e históricamente construidos (Jordan, 
1981; Lavery, 2007). Otra perspectiva del discurso de la filosofía es la denominada científica o experimental (Kauppinen, 2007; Knobe, 2004; Stuhr, 2015; Machery, 2016). En este discurso se identifican recursos cuantitativos y de ciencia cognitiva para llevar adelante la reflexión, en oposición al discurso analítico basado en la clásica intuición personal. Para Mendieta (2014), en tanto, existe otra línea de producción y desarrollo de la filosofía en la que algunos textos más personales y subjetivos, tales como los diarios, cuadernos y cartas, cumplen un rol indispensable.

Por su parte, el discurso académico y profesional de la economía y los negocios ha recibido mayor atención en comparación a otros ámbitos disciplinares (McCloskey 1983; Klamer, 1990; Samuels, 1990; Bondi, 1999; Van Hooft, 2004; Smart, 2006; Bargiela-Chiappini, Nickerson y Planken, 2013; Parodi, Boudon y Julio, 2014; Parodi, 2014; Vásquez-Rocca y Parodi, 2015; Navarro, 2015; Breeze, 2015). El discurso de la economía, que originalmente proviene de las ciencias sociales (Arrese, 2006), ha enfrentado, no obstante, un cambio en el modo de construir los significados disciplinares. En particular, ello ha ocurrido en el modo escrito, pasando desde un estilo más literario o narrativo hacia uno más técnico y matemático, en lo que se ha llamado el 'giro retórico' de la economía (McCloskey, 1983, 1994). Según esta evolución con carácter positivo, los profesionales de la economía y los negocios discuten, argumentan y persuaden con recursos propios del discurso objetivo científico, poniendo énfasis en las evidencias empíricas y las pruebas cuantificables expresadas no solo por medio del sistema verbal, sino principalmente mediante fórmulas, gráficos y tablas (Parodi, Julio y Vásquez-Rocca, 2015; Vásquez-Rocca y Parodi, 2015; Vásquez-Rocca, 2016; Figueroa, 2016; González y Burdiles, 2018).

\subsection{Las encuestas de hábitos lectores}

En las últimas décadas el interés por conocer los hábitos lectores se ha incrementado. Instituciones internacionales como las Naciones Unidas y la Organización para la Cooperación y el Desarrollo Económico han incentivado en los gobiernos y organismos privados la preocupación por generar una población lectora que se informe y pueda tomar decisiones desde una postura crítica. En este contexto, distintos países han llevado a cabo mediciones periódicas sobre el consumo lector, así como sobre preferencias lectoras y el consumo cultural en general. Específicamente, las encuestas de hábitos lectores se centran en identificar en qué formato se prefiere el libro (papel, digital o audio), qué tipos de textos son los más leídos (libros, revistas, periódicos), como también medir la lectura per capita del país y sus regiones. 
En EEUU, las encuestas muestran que el tipo de texto más leído es el libro (79\%), y el formato preferido para su lectura es el papel (65\%) (Perrin, 2016). En México, por su parte, las encuestas también señalan al libro (56\%) por sobre otros tipos de textos y también se indicó una preferencia por el formato en papel. Asimismo, se constató que cada persona declaraba leer en promedio 2,9 libros anualmente (Consejo Nacional de la Cultura y las Artes, 2006). En España se han realizado diversos estudios sobre los hábitos lectores de la población. Todos ellos han llegado a similares conclusiones: los españoles leen poco y su afición, alta en los primeros años escolares, disminuye con la edad (Ministerio de Educación, Cultura y Deporte, 2000, 2001). En Chile, el 92\% de la población informó que tiene en sus hogares entre 26 y 50 libros (Departamento de Economía, 2011). En otra encuesta también en Chile, el 41\% de la población afirmó que durante el 2014 leyó al menos un libro, mientras que el 53,9\% respondió que no leyó ninguno (Consejo Nacional de la Cultura y las Artes, 2014).

Resulta evidente que no todas las encuestas consideran los mismos criterios e indicadores, ya sea por diferentes focos de información requerida por las instituciones gubernamentales o por intereses económicos en conocer las tendencias de consumo de los lectores con el fin de tomar decisiones editoriales. En todas estas encuestas se aprecia mucho interés, por un lado, en datos demográficos, tales como edad, sexo, región o área del país en que se reside, nivel socioeconómico y, por otro, en cuestiones como lugar en donde se lee, asistencia a bibliotecas públicas o privadas y número de libros comprados, regalados o leídos. Ellas muestran menor desarrollo o finura en variables lingüísticas, textuales, genéricas o de procesamiento psicosociolingüístico. Junto a ello, las encuestas de hábitos lectores o también llamadas de comportamiento lector han centrado mayoritariamente su interés en la asiduidad de lectura del formato 'libro', muchas veces sin mucha claridad acerca de qué significa exactamente este posible género discursivo.

En nuestra revisión de la literatura y en cuanto a las encuestas disponibles, no se registra foco en los propósitos u objetivos de lectura que guían un tipo de lectura de otro. Tampoco se aprecia foco en la participación activa del lector en cuanto a sus preferencias respecto a niveles o grados de comprensión, aprendizaje y memoria o recuerdo. Una excepción son los trabajos de Baron (2015) con muestras de lectores norteamericanos, alemanes y japoneses (Baron, 2015).

\subsection{Hábitos lectores en la generación Google y en la generación Gutenberg}

Desde la invención de la imprenta en el siglo XV, los objetos de lectura fueron principalmente textos escritos en soporte papel. Este uso generalizado parece enfrentar un nuevo escenario desde que la aparición y masificación de las tecnologías de la información han supuesto modificaciones en los hábitos lectores de las 
personas. Así, en contextos educativos surgen diversas caracterizaciones de grupos humanos según su relación con las nuevas tecnologías. En la literatura de 1991 a 2014 se identifican al menos 48 términos distintos para nombrar a los usuarios de tecnología digital (Gallardo, Marqués, Bullen y Strijbos, 2015). Una de las distinciones más difundidas, aunque no exenta de polémica, es la que surge a fines de los años noventa y que diferencia entre nativos e inmigrantes digitales, basándose en los intervalos de nacimiento de los sujetos adscritos a cada generación y asociando este dato a una particular relación con el mundo digital. Los nativos digitales serían los jóvenes que, nacidos en la década del noventa, constituyen la primera generación de la revolución tecnológica, rodeada de artefactos propios de la era digital (Prensky, 2001, 2009). Serían inmigrantes digitales o pertenecientes a la 'Generación Gutenberg' (Parodi et al., en prensa), en tanto, quienes no crecieron familiarizados con estas tecnologías y debieron aprender nuevas culturas y formas de comunicación para incorporarse al mundo digital.

Un criterio categorizador algo diferente, aunque igualmente controvertido, subraya las prácticas tecnológicas que se pusieron de moda para ciertos usuarios. Es así que surge la llamada 'Generación Google', en la que se identifica a aquellos jóvenes nacidos a partir del año 1993 que viven en un mundo de conectividad permanente, puesto que utilizan internet como única fuente de información y tienen a Google como su principal motor de búsqueda (Rowlands et al., 2008; Gunter, Rowlands y Nicholas, 2009; Nicholas, Rowlands, Clark y Williams, 2011).

Aunque los términos nativo e inmigrante digital se han empleado con cierta regularidad, ha existido un considerable debate a raíz de que mucho de lo que se afirmó antes del 2008 acerca de los nativos digitales y de las implicaciones educativas de sus características carecía de evidencia empírica (Bullen, Morgan y Qayyum, 2011). Algunos estudios han avanzado en demostrar que no existen diferencias fundamentales entre nativos e inmigrantes digitales (e.g., Kennedy, Dalgarno, Bennett, Judd, Gray y Chang, 2008; Corrin, Lockyer y Bennett, 2010; Salajan, Schönwetter y Cleghorn, 2010) y que dichas divergencias podrían explicarse, más que por la edad, por la experiencia, el acceso y la oportunidad que las personas han tenido para usar las tecnologías (Brown y Czerniewicz, 2010; Czerniewicz y Brown, 2010). Desde esta misma perspectiva crítica, Selwyn (2009: 370) afirma que "whist often compelling and persuasive, the overall tenor of these discursive constructions of young people and technology tends towards exaggeration and inconsistency". En esta línea, según Selwyn (2009), muchos jóvenes hacen uso de las tecnologías digitales, pero se limitan, por ejemplo, a los juegos, la mensajería de texto y búsqueda y la recuperación de contenido en línea.

Resulta prudente, entonces, no confundir el uso frecuente y el apego a la tecnología digital con un dominio avanzado de habilidades en comunicaciones y manejo de sistemas digitales, ni menos con el desempeño y altos niveles en competen- 
cias de lectura y escritura en soportes electrónicos. Por consiguiente, no resultan del todo útiles las generalizaciones basadas en aparentes diferencias generacionales para las discusiones relativas a las características de los usuarios (Gallardo et al., 2015); tampoco lo son las descripciones incompletas o los mitos, como los que rodean a la denominada 'Generación Google' (Rowlands et al., 2008; Nicholas et al., 2011; Parodi et al., en prensa).

En este escenario, se hace imprescindible orientar adecuadamente el debate y llevar a cabo investigaciones científicas objetivas que comprueben con datos empíricos las aseveraciones que circulan desde entornos no profesionales e inexpertos (Bennett, Maton y Kervin, 2008; Ito et al., 2008; Selwyn, 2009). Al respecto, Ito et al. (2008: 4) concluyen acertadamente en que deberíamos estar "wary of the claims that a digital generation is overthrowing culture and knowledge as we know it and that its members are engaging in a new media in ways radically different from those of older generations".

En definitiva, existen otras variables contextuales que pueden explicar las diferencias en las formas de utilizar las tecnologías de la información (Jones, Ramanau, Cross y Healing, 2010). Este campo de investigación aún cuenta con un panorama empírico preliminar, que requiere avanzar a nuevas explicaciones, tales como la incidencia que tienen los propósitos perseguidos por lectores de géneros específicos en diversos formatos (papel y digital) y en variados dispositivos (Putro y Lee, 2017; Parodi et al., en prensa).

\section{MARCO METODOLÓGICO}

En lo que sigue, damos cuenta de los participantes, los instrumentos y los procedimientos del presente estudio. Cabe señalar que los instrumentos empleados en esta investigación fueron una encuesta de Hábitos de Lectura guiados por Propósitos (HALE\&P) y una entrevista semiestructurada a profesionales.

\subsection{Primer instrumento, sujetos y procedimientos}

\subsubsection{Participantes: muestra de estudiantes universitarios}

Dado que el foco de interés del Proyecto FONDECYT 1170623 es el estudio del discurso escrito de la filosofía y la economía y los negocios, para la presente investigación se empleó parte de la muestra de estudiantes universitarios seleccionados por Parodi et al. (en prensa). De esta forma, la muestra se constituyó a partir de estudiantes de cursos superiores pertenecientes a: ciencias humanas (pedagogía 
en filosofía) y ciencias económicas y negocios (ingeniería comercial y comercio), en dos universidades regionales chilenas. Este número de participantes fue estratificado de acuerdo a las siguientes variables: a) área de estudio, b) institución, c) carrera, y d) sexo. Este ejercicio dio como resultado la subdivisión mostrada en la Tabla I. Basados en los parámetros descritos, la muestra se constituyó por 580 participantes y fue diseñada considerando una proporción equitativa entre mujeres y hombres. La edad promedio de los sujetos encuestados fue de 21 años (DS 2,5). La Tabla I muestra la distribución por carrera.

Tabla I. Constitución de la muestra de estudiantes universitarios.

\begin{tabular}{|c|c|c|}
\hline Ciencias Humanas & \multicolumn{2}{|c|}{ Ciencias Económicas y Negocios } \\
\hline Filosofía & Ingeniería Comercial & Comercio \\
\hline 44 & 366 & 170 \\
\hline \multicolumn{2}{|c|}{580} \\
\hline
\end{tabular}

\subsubsection{La encuesta HALE\&P}

El objetivo de la encuesta Hábitos de Lectura guiados por Propósitos (HALE\&P) es conocer los hábitos de lectura de textos en diversos soportes y dispositivos, así como identificar géneros discursivos asociados y sus rasgos multisemióticos, todo enmarcado desde tres propósitos de lectura: a) lectura de textos académicos, b) lectura por entretenimiento, y c) lectura para buscar información. En términos generales, nos motivó la idea de identificar si los lectores establecían diferencias en sus hábitos de lectura ante diferentes soportes y dispositivos, al considerar diversos propósitos de lectura tal como la lectura académica.

La encuesta HALE\&P se compone de veinticuatro preguntas (abiertas y cerradas), distribuidas en seis dimensiones. La Tabla II da cuenta de las seis secciones, las dimensiones implicadas, los tres propósitos de lectura transversales a las preguntas y su distribución por número de preguntas. 
Tabla II. Constitución de la encuesta HALE\&P.

\begin{tabular}{|c|c|c|c|}
\hline Sección & Dimensión & $\begin{array}{l}\text { Propósitos } \\
\text { de lectura } \\
\text { (transversal) }\end{array}$ & $\begin{array}{l}\text { Número } \\
\text { de } \\
\text { preguntas }\end{array}$ \\
\hline $\begin{array}{l}\text { Parte 1: Soporte de } \\
\text { preferencia y concen- } \\
\text { tración }\end{array}$ & $\begin{array}{l}\text { Medio en el cual en general se } \\
\text { realiza la lectura: papel o digital; y } \\
\text { soporte de preferencia para man- } \\
\text { tener concentración según cada } \\
\text { objetivo de lectura. }\end{array}$ & \multirow{3}{*}{$\begin{array}{l}\text { Se considera- } \\
\text { ron } 3 \text { propósi- } \\
\text { tos de lectura: } \\
\text { Lectura acadé- } \\
\text { mica } \\
\text { Lectura por } \\
\text { entretención }\end{array}$} & 6 \\
\hline Parte 2: Dispositivo & $\begin{array}{l}\text { Dispositivo o aparato utilizado } \\
\text { para leer: teléfono, computador, } \\
\text { tablet, papel, etc. }\end{array}$ & & 2 \\
\hline $\begin{array}{l}\text { Parte 3: Género del } \\
\text { discurso }\end{array}$ & $\begin{array}{l}\text { Tipos de texto que se leen según } \\
\text { soporte }\end{array}$ & & 3 \\
\hline $\begin{array}{l}\text { Parte 4: Compren- } \\
\text { sión, memoria y } \\
\text { aprendizaje }\end{array}$ & $\begin{array}{l}\text { Procesamiento psicolingüístico } \\
\text { de la lectura: mejores resultados } \\
\text { en comprender, memorizar y } \\
\text { aprender según soporte }\end{array}$ & \multirow[t]{3}{*}{$\begin{array}{l}\text { Lectura por } \\
\text { búsqueda de } \\
\text { información }\end{array}$} & 3 \\
\hline $\begin{array}{l}\text { Parte 5: Múltiples } \\
\text { sistemas semióticos }\end{array}$ & $\begin{array}{l}\text { Rasgos de los textos: palabras, } \\
\text { gráficos, tablas, esquemas, etc. }\end{array}$ & & 4 \\
\hline $\begin{array}{l}\text { Parte 6: Costo y me- } \\
\text { dio ambiente }\end{array}$ & $\begin{array}{l}\text { Costo económico asociado al so- } \\
\text { porte de lectura e impacto sobre } \\
\text { el medio ambiente }\end{array}$ & & 6 \\
\hline
\end{tabular}

La encuesta HALE\&P fue desarrollada por los investigadores del Proyecto FONDECYT 1170623. Para su construcción se tomó en cuenta tres fuentes concurrentes y complementarias de información:

a. consulta a un grupo de tres especialistas acerca de su formato, contenidos y tipos de preguntas,

b. aplicación piloto a una muestra de participantes de las mismas carreras, pero de instituciones universitarias diferentes a las consideradas por el estudio, y

c. entrevistas a estudiantes también de instituciones diferentes a las de la muestra meta.

Basados en la información recopilada a partir de estas tres fuentes, en la versión final de la encuesta se introdujeron ajustes relativos a redacción, formato y terminología. 


\subsubsection{Procedimientos}

Tal como se indicó en la sección anterior, la encuesta se aplicó aleatoriamente a 580 estudiantes universitarios. La aleatorización se aseguró por medio de un programa computacional que seleccionaba números al azar de la lista de cada curso en los que se aplicó la encuesta. Las encuestas fueron aplicadas presencialmente y en formato papel en las salas de clase de las carreras seleccionadas por seis encuestadores previamente entrenados. Se optó por esta estrategia de aplicación, ya que otorga un mejor control de las características definidas para la muestra (carrera, sexo), asegura la obtención de una respuesta inmediata in situ por parte de los encuestados y su aplicación resulta más rápida en comparación con otros métodos, por ejemplo, usando encuestas en línea y solicitadas por correo electrónico.

Antes de la aplicación de la encuesta, a los estudiantes se les entregó un consentimiento informado en el que se les indicaba que su participación era voluntaria y que los datos entregados eran anónimos y confidenciales. La aplicación de la encuesta tomó, en promedio, 15 a 20 minutos. Las encuestas contestadas luego fueron codificadas en una planilla de datos por un grupo de codificadores entrenados por los investigadores del proyecto principal. Los análisis preliminares fueron revisados en sesiones de trabajo grupal y discutidos por todo el equipo de investigación.

\subsection{Segundo instrumento: participantes y procedimientos}

\subsubsection{Participantes: muestra de profesionales}

Los profesionales para la muestra fueron seleccionados considerando los siguientes criterios: a) contar con, al menos, 5 años de experiencia profesional, b) haber obtenido o estar cursando un programa de postgrado (magíster o doctorado) en el área en el que se desempeñan profesionalmente, $c$ ) tener publicaciones que acreditaran su desempeño como profesionales expertos de su disciplina.

La muestra de profesionales se recolectó a través de dos técnicas usadas complementariamente con el objetivo de aumentar y diversificar el grupo de profesionales. A través de un muestreo intencionado se llevó a cabo el primer acercamiento o contacto con un conjunto de profesionales de las áreas bajo estudio en una serie de instituciones públicas y privadas. En esta primera instancia se contactó a profesionales que se desempeñaban en el área de economía y negocios y de filosofía en instituciones tales como el Banco Central, entidades financieras, universidades, fundaciones, empresas y centros de investigación.

Luego de entrevistar a un primer grupo de profesionales que aceptaron par- 
ticipar en la investigación, se utilizó la técnica 'muestreo bola de nieve' (Chain Referral Sampling: Biernacki y Waldorf, 1981). Este método consiste en solicitar a los participantes entrevistados recomendar otros profesionales de la misma área disciplinar. Se les pidió a los entrevistados que diversificaran los contactos otorgados de modo de no incorporar a la muestra profesionales que se desempeñaran en los mismos lugares de trabajo. Todo esto con la intención de obtener una muestra amplia y heterogénea de profesionales en cada una de las disciplinas.

De esta forma, se conformó una muestra de 46 profesionales, 22 que ejercían labores en el área de la economía y los negocios y 24 que se desempeñaban como profesionales en el área de la filosofía. La edad promedio de los profesionales entrevistados fue de 51,4 años (DS 12,5). Esta DS obedece a que se incluyó a sujetos de un amplio rango de edad, pues la edad en sí misma no fue un factor interviniente, sino más bien el grado de experiencia y prestigio en el área. Al momento de la entrevista, todos los entrevistados firmaron un consentimiento informado en el que se les indicaba que su participación era voluntaria y que los datos entregados eran anónimos y confidenciales.

\subsubsection{La entrevista}

La entrevista tenía como objetivo principal conocer los hábitos y prácticas de lectura de profesionales en las disciplinas de filosofía y de economía y negocios, y se diseñó de modo semiestructurado a partir de un conjunto base de 10 preguntas guía, las cuales constituían la información básica y mínima que se debía abordar. Específicamente, la entrevista buscaba obtener información relativa a: 1) los géneros discursivos que leen estos profesionales en su vida laboral, y 2) el formato (papel y digital) de preferencia para la lectura de estos géneros; al mismo tiempo, se solicitaron ejemplares representativos de los géneros identificados.

\subsubsection{Procedimientos}

La entrevista semiestructurada se llevó a cabo en el lugar de trabajo de cada participante, previo contacto por correo electrónico y por teléfono. Estas entrevistas fueron realizadas por personal entrenado del proyecto y, para cuidar que se realizaran de forma homogénea entre participantes, se diseñó un protocolo de aplicación, esto es, un instrumento con foco en las preguntas y los temas objeto de investigación. Cada entrevista tomó aproximadamente entre 40 a 50 minutos. Las sesiones fueron audiograbadas y luego transcritas a una planilla de registros para codificar las respuestas. Esta transcripción se realizó siguiendo una pauta de análisis que se centraba en las preguntas base de la entrevista. Una parte importante de dichas preguntas eran de carácter cerrado o dicotómico, lo que no requería interpretación por parte de los codificadores; no obstante ello, con el fin de asegurar la mejor 
fiabilidad de los datos, todos los análisis preliminares fueron revisados y discutidos en sesiones de trabajo grupal con participación de todo el equipo de investigación.

\section{RESULTADOS Y DISCUSIÓN}

Tal como se indicó en la introducción, en este estudio nos interesa describir los hábitos lectores de una muestra de estudiantes universitarios y de un grupo de profesionales que se desempeñan en diversos contextos laborales, ambos desde dos áreas disciplinares: filosofía y economía y negocios. El objetivo final es conocer y comparar sus hábitos de lectura y prácticas lectoras respecto del soporte papel y digital, así como conocer los géneros discursivos que declaran leer con propósitos académicos o de estudio.

En lo que sigue, entregamos, en primer lugar, parte de los resultados de la encuesta a estudiantes universitarios; posteriormente, revisamos los hallazgos reportados a partir de la entrevista al grupo de profesionales. Por último, nos concentramos en la identificación, descripción y comparación de los géneros del discurso que tanto estudiantes como profesionales declaran emplear con propósitos de estudio, desde sus entornos académicos y laborales, respectivamente.

\subsection{Los resultados de la encuesta HALE\&P en estudiantes universitarios de filosofía y economía y negocios}

Cabe señalar que los resultados aportados en esta sección constituyen solo una parte del total de la información obtenida en la encuesta. Parodi et al. (en prensa) han abordado otras de las dimensiones de la encuesta considerando una muestra más amplia y diversa de sujetos en cinco áreas disciplinares.

Ahora bien, los resultados aquí analizados consideran a los 580 estudiantes universitarios, a quienes se les consultó por: el soporte general de preferencia, el mayor grado de concentración, la mejor comprensión, la mejor memorización y la mejor calidad de aprendizaje, siempre distinguiendo entre soporte papel y soporte digital. Todo ello, en el contexto de una lectura con propósito de académico. 


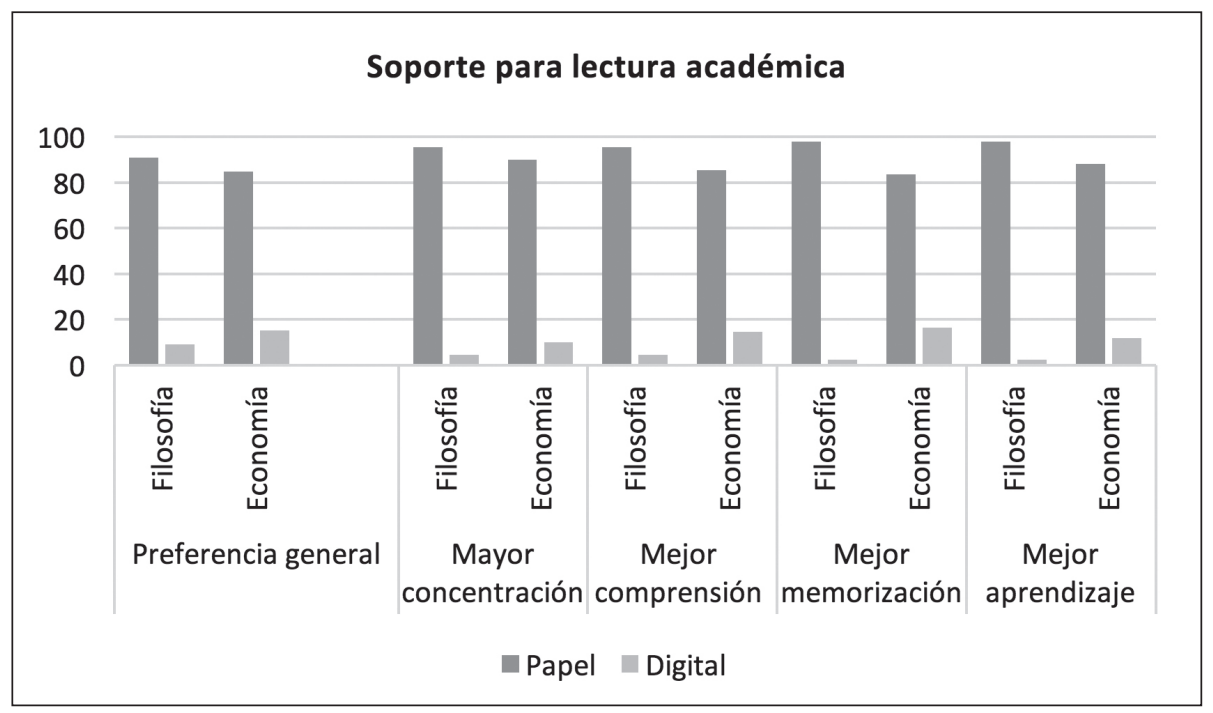

Gráfico 1. Propósito de lectura académica: preferencia general, concentración, comprensión, memorización y aprendizaje.

Ante el propósito señalado, los estudiantes de filosofía (91\%) y de economía y negocios (85\%) mayoritariamente declaran preferir el formato papel como soporte general de preferencia. Lo mismo sucede, incluso de modo levemente superior, cuando se les pregunta por el soporte en que les resulta más fácil mantener la concentración (filosofía: 95\%; economía y negocios: 90\%). Similares resultados a favor de la lectura en papel se observan en cuanto a mejor comprensión, mejor memorización y mejor aprendizaje. Esto revela, a partir de los datos de los sujetos de la muestra, un panorama altamente homogéneo y se registra -en todos los casos- un resultado por sobre el $85 \%$ a favor de una lectura en soporte papel. Como se desprende, los hallazgos revelan que la disciplinariedad no incide en los hábitos lectores de este grupo de estudiantes; sin importar la procedencia, todos los lectores de la muestra se inclinan claramente por el formato papel para tareas académicas (todas las diferencias son estadísticamente significativas, ver Anexo 1).

Cabe destacar que estos hallazgos concuerdan con los de otras investigaciones que registran hábitos de lectura en estudiantes universitarios de diversas nacionalidades, entre otros, japoneses, turcos, españoles, chinos, chinos malayos, alemanes y estadounidenses (e.g., Woody, Daniel y Baker, 2010; Jafre, Pour-Mohammadi y Choon, 2011; Mangen, Walgermo y Bronnick, 2013; Baron, 2015; Kazanci, 2015; Wang y Bai, 2016). Del mismo modo, ello también acontece en otras áreas disciplinares en estudiantes chilenos, tales como historia y castellano (Parodi et al., en prensa). En todos estos casos, la tendencia registrada privilegia sistemáticamente el formato papel. 


\subsection{Resultados de las entrevistas a profesionales de filosofía y economía y negocios}

Tal como se indicó en las secciones previas, ha existido un lento pero progresivo interés en estudiar el discurso profesional y sus variaciones disciplinares, aunque mucha de la investigación se concentra en estudios descriptivos centrados en los rasgos de los textos escritos. Desde esta mirada, en este apartado presentamos -en un primer momento- los resultados generales respecto de los hábitos declarados de lectura a partir de las entrevistas a cuarenta y seis profesionales de filosofía y de economía y negocios. El Gráfico 2 resume los datos porcentuales en cuanto al soporte de preferencia (papel o digital) en el contexto de una lectura general y de una lectura con propósito de estudio.

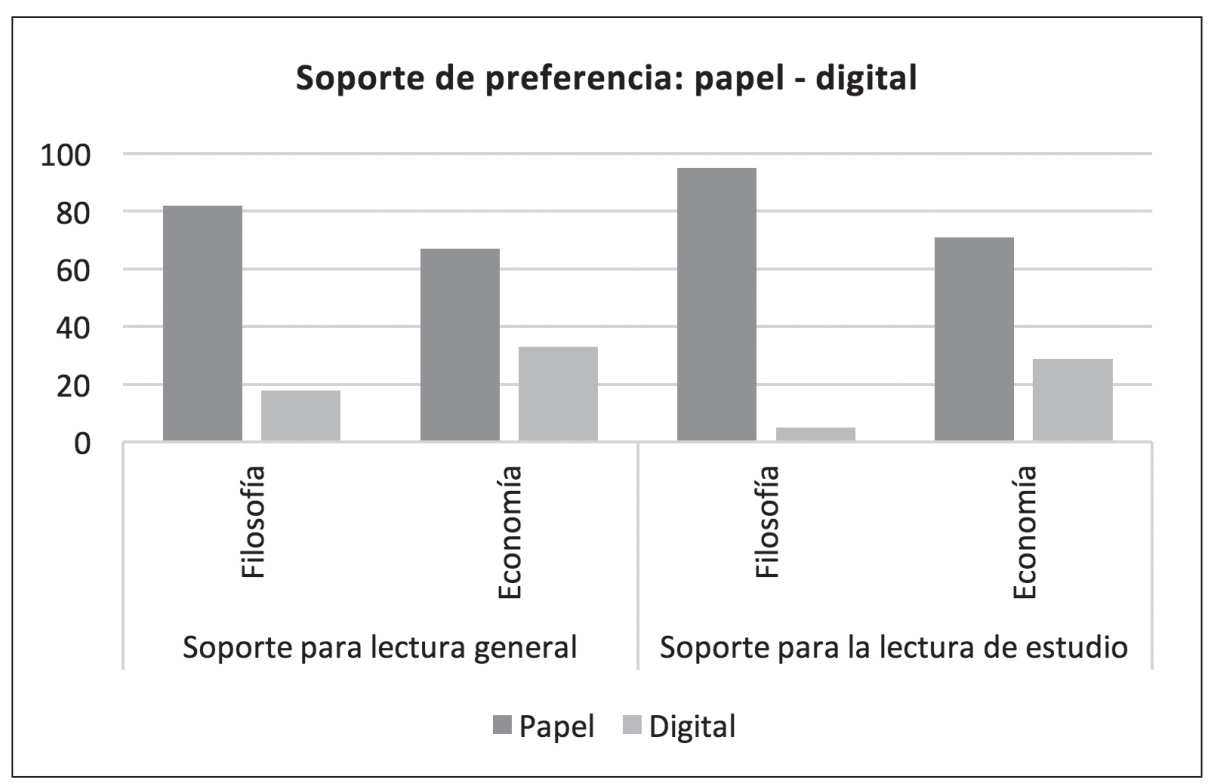

Gráfico 2. Soporte de preferencia para lectura general y lectura de estudio.

Tal como se aprecia en el gráfico, tanto los profesionales de filosofía (82\%) como los de economía y negocios (67\%) declaran preferir mayoritariamente el soporte papel para una lectura general; sin embargo, esta diferencia entre papel y digital solo es estadísticamente significativa en el caso de filosofía (ver Anexo 2). Interesantemente, cuando se les pregunta por sus prácticas de lectura, pero en un contexto de estudio, los porcentajes en favor del sustrato papel aumentan: 95\% en filosofía y $71 \%$ en economía y negocios. En este caso, todas las diferencias son es- 
tadísticamte significativas (ver Anexo 2). Como se desprende, solo uno de los veintidós profesionales entrevistados de filosofía (5\%) enuncia que opta por el soporte digital para leer cuando estudia. No obstante lo anterior, en el mismo gráfico se observa que los profesionales de economía y negocios muestran mayor tendencia hacia el formato digital que los de filosofía, aunque es -comparativamente- una tendencia menor (33 y $30 \%)$.

Estos resultados son coincidentes con los reportados en la sección anterior (ver Gráfico 1) a partir de la opinión de estudiantes universitarios de las mismas áreas disciplinares: un alto porcentaje de ellos se inclina a favor del formato papel sin que el área del conocimiento de origen revele diferencias sustanciales. Estos hallazgos indican que, sin distingo de ámbito académico o profesional y sin diferencia disciplinar, todos los encuestados y entrevistados declaran en su mayoría su preferencia por el formato papel para una lectura general y una más específica de estudio.

Comparativamente con el ámbito académico, son más escasas las investigaciones que se han enfocado en los hábitos de lectura de profesionales y que se han interesado en las experiencias y géneros de lectura en contextos laborales. Algunas de las disponibles han centrado su atención en las prácticas comunicativas de médicos y profesionales de la salud (e.g., Stinson y Mueller, 1980; Giuse, Huber, Guise, Brown, Bankowtz y Hunt, 1994; Saint et al., 2000). Otras investigaciones se han enfocado más directamente en los géneros que se leen en la práctica profesional de diversas áreas y registran el empleo de formatos papel/digital (e.g., Shaikh y Chaparro, 2004; Jafre et al., 2011; Tenopir, Volentine y King, 2012; Nathan y Grossman, 2012; Sahu y Singh, 2013; Tenopir, King, Christian y Volentine, 2015). Interesantemente, los hallazgos de investigaciones similares a la presente, en contextos profesionales anglosajones, llegan a conclusiones semejantes a las aquí reportadas en cuanto a preferencia por el formato papel para propósitos de estudio: "academics still print-out articles for final reading, although on-screen reading is increasing." (Tenopir et al., 2012: 285); incluso se señala que "e-books have not yet made much of an inroad for scholarly reading by UK academics." (Tenopir et al., 2012: 288).

Algunas de las preocupaciones recurrentes que señalan estos estudios con profesionales de diversas áreas disciplinares, interesados en qué leen, cuán a menudo lo hacen y qué géneros prefieren, dice relación con que: "To ensure that professionals maintain competency in their discipline, jurisdictions commonly require that members of the profession participate in formal continuing education (CE) activities" (Nathan y Grossman, 2012: 600). Dado que en la actualidad existe consenso respecto de la naturaleza dinámica de la formación continua, para los profesionales de todas las áreas del conocimiento, "reading on a regular basis is important, not only because it keeps the reader up-to-date with changes affecting 
the profession, but also because it exposes the reader to opinions, philosophies, arguments, theories, book reviews, advice, and even employment opportunities." (Nathan y Grossman, 2012: 600).

Ahora bien, los datos entregados hasta aquí revelan un panorama relativamente homogéneo en cuanto a hábitos y prácticas de lectura para estudiantes y profesionales de la filosofía y la economía y los negocios. En su mayoría, la evidencia aportada muestra una ostensible preferencia por el sustrato papel, cuando se trata de una lectura por estudio; en especial, ello ocurre, más marcadamente, toda vez que se busca comprender, memorizar y aprender. No parece entonces emerger diferencias radicales debidas a la mayor profesionalización de los lectores ni en cuanto a la disciplinariedad.

En lo que sigue, nos concentramos en otro foco de información, también objetivo de este artículo: los géneros del discurso escrito disciplinar. Ellos han sido identificados a partir de las encuestas a estudiantes universitarios y por medio de las entrevistas a profesionales.

\subsection{Géneros del discurso disciplinar: filosofía y economía y negocios}

En este apartado entregamos una síntesis de los géneros del discurso de la filosofía y de la economía y los negocios, la cual ha sido construida a partir de la información brindada por estudiantes y profesionales como mecanismos discursivos de lectura con propósitos académicos. Esto quiere decir que estos géneros han sido identificados, en su formato papel y digital, desde un propósito de lectura muy específico que implica, entre otros, acceso, construcción y comunicación de conocimientos disciplinares especializados. Para ser consignado como un género, bastaba que fuera mencionado una vez por estudiantes o profesionales.

Cabe destacar que, en la elaboración de esta síntesis presentada por medio de tres diagramas de Venn, se ha debido hacer un esfuerzo por aunar e integrar una amplia y diversa variación denominativa y terminológica para hacer referencia a los géneros que se leen en contextos académicos y profesionales. A modo de ejemplo, consignamos el Esquema, que también incluye el Diagrama; caso similar ocurre con Artículo y Paper. No obstante ello, destacamos el valor, tanto en las encuestas como en las entrevistas, de haber incluido preguntas abiertas acerca de los géneros y no ofrecer una lista previamente determinada y cerrada, la cual estimamos limita la posibilidad de conocer la valiosa opinión de estudiantes y profesionales.

A continuación, en esta primera figura reportamos la síntesis de géneros de filosofía. Tal como ya se ha señalado, se consignan géneros identificados para el ámbito estudiantil y para el profesional, así como la indicación de formato de lectura: papel, digital o ambos (en la intersección). 


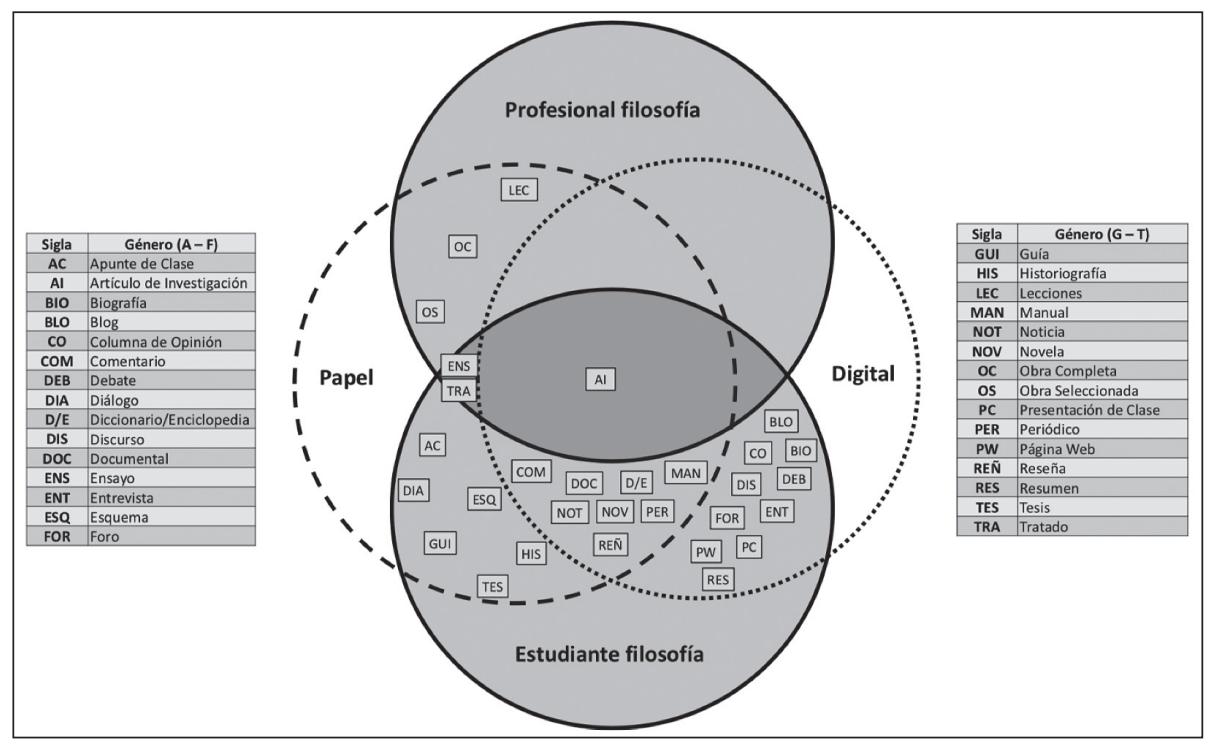

Figura 1. Géneros identificados por estudiantes universitarios y por profesionales de filosofía.

Tal como se aprecia en la Figura 1, existe un número mayor de géneros que se declara leer en la universidad, señalados por los estudiantes, en comparación con los identificados por los profesionales. Estos últimos afirman leer textos pertenecientes a 6 géneros. De ellos, 5 se leen en formato papel: Ensayo (ENS), Tratado (TRA), Lecciones (LEC), Obra Completa (OC) y Obra Seleccionada (OS). El Artículo de Investigación (AI) es el único que los profesionales leen tanto en papel como en digital, es decir, es un género dual en cuanto a soporte. Este resultado es coincidente con los datos reportados en la sección previa en que la mayoría de los profesionales de filosofía ( 21 de 22 entrevistados) declaraba que, ante un propósito de estudio, preferían el sustrato papel.

Según se observa en la misma Figura 1, de los 6 géneros profesionales, tres también son identificados por los estudiantes (ENS, TRA y AI). También en el contexto estudiantil universitario destaca la preferencia hacia géneros en formato papel en coincidencia con lo reportado por ellos mismos en las encuestas respecto a formato de preferencia para propósitos de estudio (Gráfico 1: sobre $90 \%$ de los encuestados a favor del papel). En los otros 24 géneros académicos mencionados se detecta variación entre el soporte papel y el digital, aunque la tendencia es marcadamente a favor del papel.

Interesantemente, los estudiantes de filosofía mencionaron leer la Novela como género de estudio en ambos sustratos, lo que no coincide con otras investigaciones sobre la lectura de este género, debido a que en ellas se afirma que la Novela se 
lee específicamente en papel y con el objetivo de entretenerse (Mangen y Van der Weel, 2016). Otro hallazgo que destaca fuertemente es que los géneros predilectos de la filosofía son leídos en el formato papel, como lo son las Lecciones, Obra Completa, Obra Seleccionada, Ensayo, Tratado y Diálogo. Junto a ello, y como fue mencionado en el párrafo anterior, el Ensayo, el Tratado y el Artículo de Investigación son los géneros que circulan en el ámbito académico y el profesional. En el caso del Ensayo, probablemente se emplea para aprender postulados planteados por filósofos que se encontraban en una etapa formativa (Peters, 2008). Por otro lado, el Tratado permite acceder a las reflexiones y discusiones ecuménicas (Kwak, 2010; Liceanu, 2017) y así apropiarse del conocimiento especializado de la disciplina (Lavery, 2007; Baranda, 2011). Similar al Tratado, el Artículo de Investigación opera como un (macro)género transversal de acceso al conocimiento especializado para los que están en formación y de acceso también, pero, primordialmente, de construcción y de comunicación de conocimientos para aquellos que se desempeñan como filósofos (Bolívar y Parodi, 2015; Parodi, 2015). Así, el AI reúne bajo esta etiqueta global a una variedad de géneros que se publican en revistas especializadas, pero que más específicamente podrían ser analizados como ensayos o tratados, entre otros (Herrera, 2016).

En síntesis, de la amplia gama de géneros señalados (en total 30), solo 3 son mencionados como leídos por estudiantes y profesionales. De ellos, el AI es el único identificado en los dos formatos (papel y digital). El ENS y el TRAT, géneros icónicos de la filosofía, son leídos exclusivamente en formato papel, tanto por estudiantes como por profesionales. LEC, OC y OS son géneros que destacan como representantes exclusivos del mundo profesional. Este panorama a partir de los recursos genéricos escritos en formato papel, más limitado en el mundo laboral que el universitario, revela una focalización en la construcción y transmisión del conocimiento profesional con menor variación genérica pero con alta especialización. También se identifican al ENS, al TRA y al AI como mecanismos de tránsito desde el espacio académico al profesional, pues actúan como géneros de interconexión que acercan a la universidad al contexto profesional.

A continuación, en la Figura 2, se presenta la síntesis para los géneros de lectura en estudiantes universitarios y en profesionales de economía y negocios. 


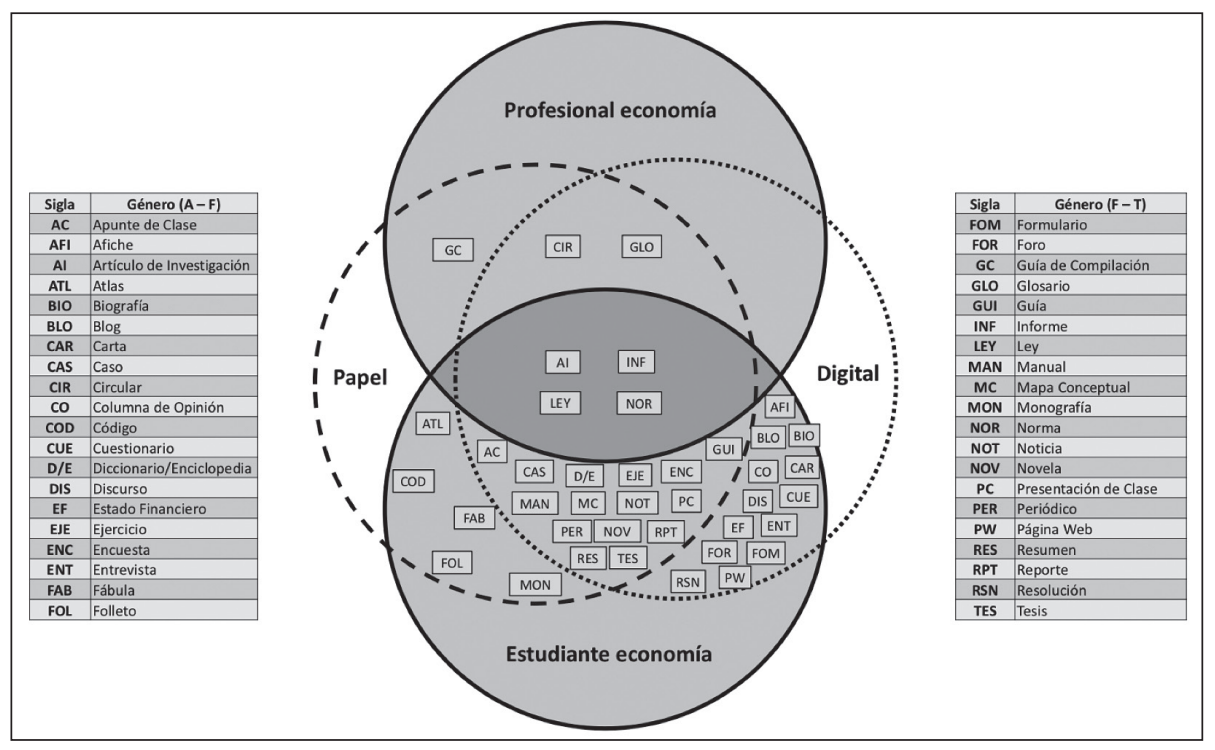

Figura 2. Géneros identificados por estudiantes universitarios y por profesionales de economía y negocios.

Los datos registrados en la Figura 2 para el discurso escrito de la economía y los negocios indican que, en total, se identifican 40 géneros para propósitos de estudio en el mundo académico y profesional. De ellos, 3 son exclusivos de los profesionales (Glosario, Guía de Compilación y Circular), 33 son exclusivos de los estudiantes universitarios y solo 4 géneros son compartidos por ambos (Artículo de Investigación, Informe, Norma y Ley). Estos datos coinciden con lo observado previamente en el dominio disciplinar de la filosofía en cuanto a una menor variedad de géneros en el ámbito profesional y a ubicar al AI como un género dual (papel/digital), leído por ambos grupos de informantes. Solo que, en el discurso de la economía y los negocios, también se aprecia esta característica dual para Informe, Norma y Ley y para ambos grupos de sujetos.

Asimismo, se aplica al AI lo mismo señalado para discurso de la filosofía en cuanto a su carácter de macrogénero. Junto a ello, es preciso señalar que el género Informe (INF) también opera como un macrogénero, dentro del cual investigaciones previas han identificado un conjunto amplio de géneros específicos: Informe de Política Monetaria (Parodi et al., 2015; Vásquez-Rocca y Parodi, 2015; Vásquez-Rocca, 2016), Informe de Empleo Regional (Oyarzún, 2016), Informe Mensual de Estadísticas Monetarias y Financieras (Molina, Vásquez-Rocca y Parodi, en prensa), Informe Anual del Fondo Monetario Internacional (Moreno-de León, 2017), e Informe Estabilidad Financiera (González y Burdiles, 2018).

Según se aprecia en la Figura 2, en lo declarado por los estudiantes de econo- 
mía y negocios en cuanto a géneros, se detecta una marcada tendencia a preferir tanto el formato digital como el dual, en contraste con el papel. Sin embargo, se debe recordar que cuando el propósito de lectura es académico y no se distingue géneros, los universitarios prefieren leer en soporte papel, tal como lo señalan los datos del Gráfico 1. Los profesionales de economía y negocios en tanto tienden a preferir géneros duales (6 de 7).

\subsection{Comparación de géneros entre estudiantes y profesionales de ambas disciplinas}

El tercer diagrama de Venn da cuenta de los géneros del discurso académico y del discurso profesional en ambos dominios disciplinares, con atención a los formatos papel y digital.

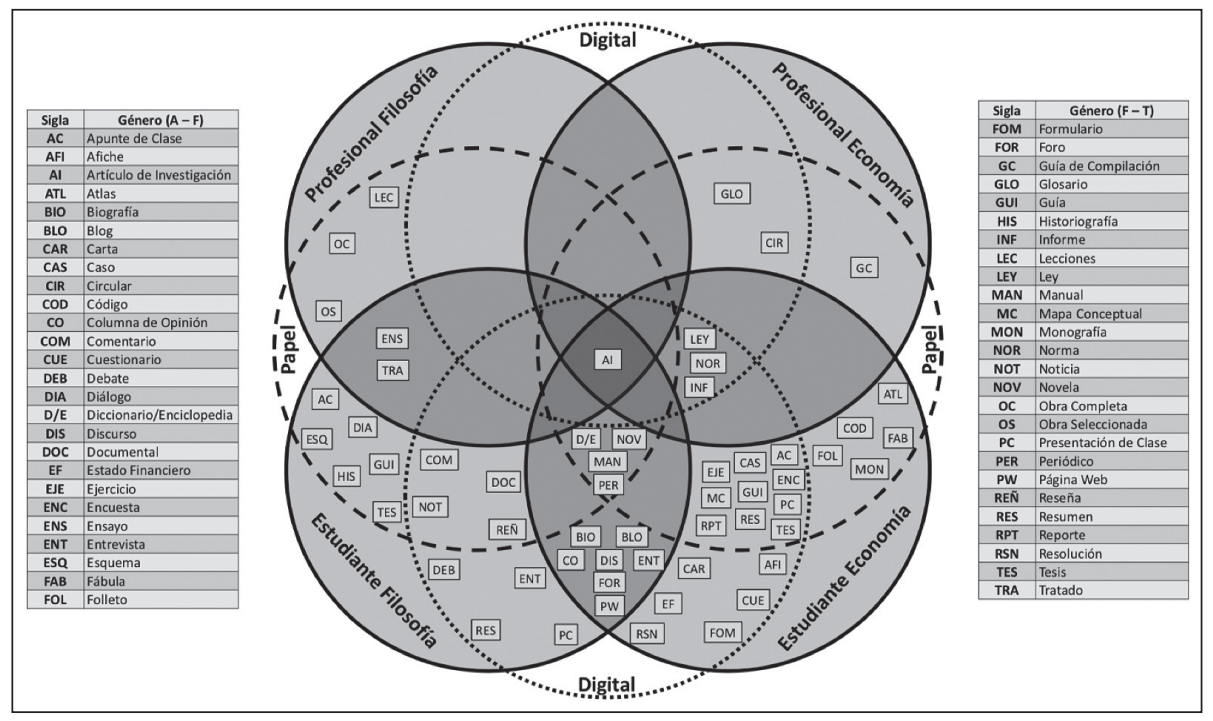

Figura 3. Géneros identificados por estudiantes universitarios y por profesionales de filosofía y de economía y negocios.

Un primer hallazgo que emerge a partir de la Figura 3 dice relación con la menor ocurrencia de géneros identificados en el discurso profesional (DP) en comparación con el discurso académico (DA), hecho coincidente en ambas áreas disciplinares en estudio. Según los géneros reportados por los profesionales de ambas disciplinas, se desprende que se concentran en solo unos géneros, los cuales son prototípicos para cada una (DP: 6 para filosofía y 7 para economía y negocios). En 
el caso del DA, se identifica una amplia gama de géneros muy heterogéneos (DA: 28 para filosofía y 36 para economía y negocios). En otras palabras, al comparar los géneros del mundo académico y el profesional, lo que surge con gran claridad a partir de estos datos es la reducción de recursos genéricos en el DP para acceder y construir el conocimiento especializado. Y ello ocurre sistemáticamente tanto en filosofía como en economía.

Entonces, lo que observamos en estos hallazgos es una atomización y especialización en algunos géneros profesionales específicos en el discurso escrito de estas dos disciplinas. En comparación al mundo académico, se detecta poca variedad genérica, pero alta especialización en la comunicación del conocimiento profesional. Al respecto, Parodi (2009), a partir de un corpus de textos de cuatro áreas disciplinares (psicología, trabajo social, química industrial e ingeniería en construcción), observó que -para estas disciplinas y con procedimientos de recolección de corpus- la diversidad de géneros que emergió desde el campo laboral se reveló más heterogénea que la que se detectó en el ámbito universitario. No obstante ello, la alta especialización genérica en el dominio profesional es un rasgo que se mantiene en ambos estudios.

Este primer hallazgo también pone de relieve las coincidencias de géneros académicos entre estudiantes de filosofía y economía y negocios. Tal como se adelantó más arriba, se observa una interesante heterogeneidad de géneros escritos en ambas disciplinas a nivel de grado universitario, de los cuales 12 son compartidos ( 7 en formato digital y 5 en formato dual). La mayoría de ellos muestra un carácter probablemente no especializado, entre otros: Novela, Blog, Biografía, Discurso, Columna de Opinión, Periódico, Página Web; no obstante ello, estos géneros han sido identificados en el contexto de propósitos de estudio. También se registran otros géneros compartidos en ambas disciplinas y claramente de naturaleza más disciplinar: Artículo de Investigación, Manual y Diccionario/Enciclopedia.

Como un segundo hallazgo es posible apreciar que -en general- existe una tendencia mayoritaria por géneros en formato dual, esto es, que se declara la preferencia compartida por formato tanto papel como digital, indistintamente de la disciplina de origen. Esta tendencia es más marcada en el discurso de la economía y los negocios que en el discurso de la filosofía y, de modo más específico, es liderada por los estudiantes de economía y negocios. La excepción a la tendencia dual se registra en los profesionales de filosofía, quienes prefieren mayoritariamente leer géneros en formato papel. De los seis géneros que ellos señalan, todos son en papel con la única excepción del AI que se registra en ambos formatos. En todo caso, no obstante este panorama general, cabe señalar que los estudiantes de filosofía identifican exclusivamente en formato papel a los géneros fundamentales de acceso al conocimiento altamente especializado disciplinar: Tratado y Ensayo. Como se observa, en cuanto a géneros y su sustrato, sí se detectan diferencias entre disciplinas. A la luz de estos datos, si bien se podría argumentar, en base al promedio de edad, 
que los estudiantes universitarios pertenecerían a la llamada generación Google, mientras que los profesionales integrarían la generación Gutenberg o serían considerados como inmigrantes digitales (Prensky, 2001, 2009; Rowlands et al., 2008; Gunter et al., 2009), los hallazgos aquí reportados nos conducen a sostener que tal distinción no se aplicaría en sentido estricto.

Ahora bien, cabe señalar que existe una sola excepción a la especialización genérica identificada por disciplina y esta la constituye el AI, que emerge como el único género que ocurre en ambos dominios disciplinares, tanto en el DA como en el DP. En este punto existe una cuestión relevante que señalar: se debe precisar que este hallazgo podría no resultar en un grado de alta homogeneidad o comunalidad genérica entre disciplinas. No conocemos en detalle las características de los AIs de una y otra área y es probable que exista, al mismo tiempo, variación genérica tanto entre disciplinas como al interior de una misma disciplina. En otras palabras, un género como el denominado AI puede resultar ser una categoría amplia e inclusiva y operar como un macrogénero, el cual acoja una amplia variación, tal como se ha comprobado ocurrir, por ejemplo, con el género Manual entre las áreas de ciencias básicas y ciencias sociales y humanas (Parodi, 2015). Ciertamente, existirá variación temática y conceptual, pero muy probablemente exista variación retórico-funcional organizacional y metodológica. Un AI de econometría presenta rasgos muy diversos a un AI que da cuenta de una reflexión teórica sobre la filosofía postmoderna o sobre la filosofía experimental (Kauppinen, 2007). Al respecto, Herrera (2016:8) señala, para el caso de filosofía, que “...los artículos de investigación de filosofía inscriptos en diferentes perspectivas filosóficas presentan especificidades intradisciplinares tales que no es posible caracterizarlos de modo generalizado".

Un asunto que debe ser considerado al momento de analizar los géneros que circulan en los mundos académicos y profesionales es que las demandas cognitivas de unos y otros géneros muy probablemente son bastante diferentes. Es muy posible que leer en el mundo académico universitario para los estudiantes en formación imponga ciertas demandas que en el mundo laboral sean diferentes. Incluso, se hace evidente que un mismo género puede ser leído con demandas incrementales en un contexto $\mathrm{u}$ otro, o para un propósito $\mathrm{u}$ otro. Leer una Norma para buscar y reportar un dato numérico específico no es lo mismo que leerla para comprender sus implicancias en la política económica actual o futura. Mucho más complejo resulta la lectura de este mismo género si debe ser comprendido e integrado con información de otras fuentes y géneros en la búsqueda del significado ('search after meaning', Graesser, Singer y Trabasso, 1994) para alcanzar así la construcción de una representación mental compleja e integradora, todo lo cual en su conjunto impone compatibilizar diversas organizaciones retóricas y variados dispositivos más argumentativos o más explicativos como pueden ser, por ejemplo, los del Informe Mensual de Estadísticas Financieras o 


\section{del Informe Anual del Fondo Monetario Internacional.}

Entonces, no solo es relevante la identificación de un género en un determinado contexto académico o profesional, sino que se debe contemplar la tarea en la cual se encuentra incrustado y las demandas cognitivas particulares que efectivamente se ejecutan en respuesta a propósitos de lectura situados. En la actualidad aún es escasa la información disponible en estos y otros asuntos relacionados en textos en español (e.g., Parodi y Julio, 2015, 2016, 2017; Parodi, Julio y Recio, 2018, Parodi, Julio, Nadal, Burdiles y Cruz, 2018).

\section{CONCLUSIONES}

Hasta donde hemos podido comprobar, son escasas las investigaciones previas que indagan, en conjunto, hábitos lectores de profesionales y estudiantes universitarios de diversas disciplinas, preferencias de formato (papel y digital), propósitos de lectura y distinción de géneros. Entonces, este sería uno de los primeros acercamientos a conocer, comparativamente, qué declaran estudiantes y profesionales expertos respecto de sus prácticas lectoras en filosofía y economía y negocios.

Los principales hallazgos indican que:

1) Independientemente de los géneros, tanto estudiantes como profesionales de filosofía y economía y negocios declaran una categórica preferencia por el formato papel para tareas de estudio y aprendizaje.

2) Con atención a los géneros escritos para propósitos de estudio, por una parte, se identifica una contundente preferencia por géneros en formato papel por parte de los profesionales en filosofía y economía y negocios. Por otra, se constata que los estudiantes universitarios, sin importar la disciplina de procedencia, optan preferentemente por géneros duales (papel/digital); en menor medida destaca la identificación a favor de géneros únicamente en digital y, por último, solo en formato papel.

3) Respecto de la variedad de géneros identificados, es relevante la atomización y especialización en géneros profesionales, en comparación con la mayor heterogeneidad de géneros académicos. En suma, en el discurso profesional de ambas disciplinas se detecta escasa variedad genérica, pero alto grado de especialización.

4) Los géneros que construyen y comunican el discurso disciplinar (tanto académico como profesional) de filosofía se identifican en formato papel (Tratado y Ensayo). Por su parte, los géneros altamente especializados de economía y negocios se constatan en formato dual (papel/digital): Informes, Norma y Ley. Ello pone de relieve que, en los géneros altamente especializados, se detecta una variación disciplinar respecto del sustrato de preferencia. 
En resumen, los resultados indican que los encuestados y entrevistados muestran disponer de un conocimiento acerca del discurso escrito y respecto de procesos lectores para propósitos de estudio y aprendizaje, tanto en ámbitos disciplinares universitarios como laborales. Los sujetos de ambas muestras revelan, a través de sus respuestas, conciencia y conocimiento de los mecanismos discursivos de acceso al conocimiento especializado y ofrecen una amplia y diversa gama de géneros al ser consultados por los textos que leen cuando estudian.

Los datos recabados permiten afirmar que, en los sujetos encuestados y entrevistados, existe una tendencia importante a preferir el formato papel, tanto en el discurso académico como en el profesional, para tareas de estudio y aprendizaje. En este sentido, es posible que esta tendencia revele un mayor vínculo entre la función epistémica de la lectura y el sustrato papel, esto es, que los lectores asocien al papel un mayor y mejor acceso a la construcción de conocimientos disciplinares. Este mejor y mayor acceso se basaría en que este medio resulta más tangible, táctil, kinésico y corpóreo, todo lo que generaría una percepción y dimensionamiento del espacio físico diferente, más propicio para la lectura detenida, reflexiva y de aprendizaje. Incluso es probable que el sustrato papel favorezca una mayor conexión afectiva entre el lector y el texto. También es posible que los lectores perciban el formato papel con cierta continuidad estructural y que logren visualizar de mejor modo el todo (principio gestáltico de continuidad) al tener entre sus manos un libro o un artículo que dimensionan en su extensión, es decir, logran percibir los límites de un texto en papel. Mientras que en el formato digital, muchas veces, los textos electrónicos se presentan como documentos infinitos cuyas fronteras y límites no resultan fáciles de determinar (provocando la sensación del denominado 'efecto tunel': por momentos no se sabe exactamente dónde se está ni se logra ver el final). De hecho, la tecnología digital ha buscado emular algunos de los rasgos del sustrato papel en los dispositivos electrónicos, intentando capturar y trasmitir algunos de sus beneficios y fortalezas.

Por último, cabe señalar que un estudio de esta naturaleza enfrenta algunas limitaciones inherentes a la investigación de hábitos declarados. Por un lado, los resultados corresponden a lo que manifiestan los encuestados y entrevistados y no necesariamente a lo que puedan efectivamente llevar a cabo en sus contextos universitarios y laborales. Por otro, las muestras de estudiantes y profesionales se determinaron mediante procedimientos diversos, fundamentalmente debido al tipo de acceso disponible divergente para contactar a uno y otro grupo de lectores. Los estudiantes universitarios son encuestados como parte de sus actividades cotidianas en donde forman grupos amplios. Y los profesionales deben ser contactados uno a uno en sus lugares de trabajo, lo que vuelve la tarea de entrevistarlos mucho más lenta y gravosa y, por consiguiente, el tamaño muestral debe valorarse con otros parámetros. No obstante lo anterior, estimamos que esta aproximación exploratoria y comparativa entre el discurso escrito académico y el profesional ofrece 
hallazgos relevantes: catastro amplio de géneros que efectivamente son declaradas por los usuarios en torno a un propósito de lectura de estudio, contraste entre los géneros académicos y profesionales en filosofía y en economía y negocios, y registro de preferencia por formato papel y digital en géneros de contextos académicos y profesionales disciplinares.

\section{REFERENCIAS}

Arnoux, Elvira, (2016). Las escrituras profesionales: dispositivos argumentativos y estrategias retóricas. Conferencia plenaria $8^{\circ}$ Congreso Cátedra UNESCO Lectura y Escritura, Universidad de Costa Rica, Costa Rica.

Arnoux, Elvira, Di Stefano, Mariana, y Pereira, Cecilia. (2010). Materiales clínicos y supervisión: escritos del campo psicoanalítico. En G. Parodi. (Ed.), Alfabetización académica y profesional en el Siglo XXI: leer y escribir desde las disciplinas (pp. 185-213). Santiago de Chile, Chile: Ariel.

Arrese, Ángel. (2006). Periodismo económico. Entre la simplificación y el rigor. Cuadernos de Información, 19, $42-49$.

Baranda, Consolación. (2011). Formas del discurso científico en el Renacimiento: tratados y diálogos. Studia Aurea, 5, 1-21.

Bargiela-Chiappini, Francesca, Nickerson, Catherine, y Planken, Brigitte. (2013). Business discourse. Nueva York, Estados Unidos: Palgrave Macmillan.

Baron, Naomi. (2015). Words Onscreen. The fate of reading in a digital world. Oxford: Oxford, England: University Press.

Bazerman, Charles, y Paradis, James. (1991). Textual dynamics of the professions: Historical and contemporary studies of writing in professional communities. Wisconsin, USA: University of Wisconsin Press.

Becher, Tony, y Trowler, Paul. (2001). Academic tribes and territories. Intellectual enquiry and the culture of disciplines. Philadelphia, USA: Open University Press.

Bennett, Sue, Maton, Karl, y Kervin, Lisa. (2008). The 'digital natives' debate: a critical review of the evidence. British Journal of Educational Technology, 39(5), 775-786.

Berkenkotter, Carol, Huckin, Thomas, y Ackerman, John. (1991). Social context and socially constructed texts: The initiation of a graduate student into a writing research community. En C. Bazerman y J. Paradis. (Eds.), Textual dynamics of the professions (pp. 191-215). Wisconsin, USA: The University of Wisconsin.

Bhatia, Vijay. (1993). Analysing genre: Language use in professional settings. Londres, Inglaterra: Longman.

Bhatia, Vijay. (2002). A Generic View of Academic Discourse. En J. Flowerdew (Ed.), Academic discourse (pp. 21- 39). Londres, Inglaterra: Pearson Education. 
Bhatia, Vijay, Garzone, Giuliana, Salvi, Rita, Tessuto, Girolamo y Williams, Christopher. (Eds.). (2014). Language and law in professional discourse. New Castle: Cambridge Scholars Publishing.

Biernacki, Patrick, y Waldorf, Dan. (1981). Snowball sampling: Problems and techniques of chain referral sampling. Sociological Methods \& Research, 10(2), 141-163.

Boden, Deirdre. (1994). The business of talk. Organizations in action. Londres, Inglaterra: Polity Press.

Bolívar, Adriana, y Parodi, Giovanni. (2015). Academic and professional discourse. En M. Lacorte (Ed.), The Routledge handbook of Hispanic applied linguistics (pp. 459-476). Londres, Inglaterra: Routledge.

Bondi, Marina. (1999). English across genres: Language variation in the discourse of Economics. Modena, Italy: Il Fiorino.

Breeze, Ruth. (2015). Corporate discourse. Londres, Inglaterra: Bloomsbury.

Brown, Cheryl, y Czerniewicz, Laura. (2010). Debunking the 'digital natives': Beyond digital apartheid, towards digital democracy. Journal of Computer Assisted Learning, 26(5), 357-369.

Bullen, Mark, Morgan, Tannis, y Qayyum, Adnan. (2011). Digital learners in higher education: Generation is not the issue. Canadian Journal of Learning and Technology, 37(1), 1-24.

Burdiles, Gina. (2012). Descripción de la organización retórica del género Caso Clínico de la Medicina. Tesis doctoral. Valparaíso, Chile: Pontificia Universidad Católica de Valparaíso.

Burdiles, Gina. (2016). Género Caso Clínico: Organización retórica de su macromovida Relato del Caso en publicaciones médicas chilenas. Revista Signos. Estudios de Lingüistica, 49(91), 192-216.

Cassany, Daniel y López, Carmen. (2010). De la Universidad al mundo laboral: Continuidad y contraste entre las prácticas letradas académicas y profesionales. En G. Parodi (Ed.), Alfabetización académica y profesional en el Siglo XXI: leer y escribir desde las disciplinas (pp. 347-374). Santiago de Chile, Chile: Ariel.

Connor, Ulla, y Upton, Thomas. (Eds.). (2004). Discourse in the professions. Perspectives from corpus linguistics. Amsterdam, Netherland: Benjamins.

Consejo Nacional de la Cultura y las Artes. (2006). Encuesta nacional de lectura. México, México: Consejo Nacional para la Cultura y las Artes.

Consejo Nacional de la Cultura y las Artes. (2014). Segunda encuesta nacional de participación y consumo cultural ENPCC. Valparaíso, Chile: Ediciones Cultura.

Corrin, Linda, Lockyer, Lori y Bennett, Sue. (2010). Technological diversity: An investigation of students' technology use in everyday life and academic study. Learning, Media and Technology, 35(4), 387-401.

Cubo de Severino, Liliana. (Coord.) (2005). Los textos de la ciencia. Córdoba, Argentina: Comunicarte. 
Czerniewicz, Laura, y Brown, Cheryl. (2010). Born into the Digital Age in the south of Africa: The reconfiguration of the 'digital citizen'. En L. DirckinckHolmfeld, V. Hodgson, C. Jones, M. de Laat, D. McConnell y T. Ryberg (Eds.), Proceedings of the 7th International Conference on Networked Learning 2010, (pp. 859-865). Aalborg, Denmark: Aalborg University.

Departamento de Economía. (2011). Estudio sobre el comportamiento lector a nivel nacional. Informe Final. Santiago de Chile, Chile: Universidad de Chile.

Figueroa, Lisette. (2016). Descripción retórico-funcional del género Boletín Informativo del área de empleo del INE. Tesis de Licenciatura en Lengua y Literatura Hispanoamericana. Valparaíso, Chile: Pontificia Universidad Católica de Valparaíso.

Flowerdew, John. (2002). Introduction: Approaches to analysis of academic discourse in English. En J. Flowerdew (Ed.), Academic discourse (pp. 1-17). Londres, Inglaterra: Longman.

Foucault, Michel. (1972). The Archaeology of knowledge. Nueva York, USA: Pantheon.

Gallardo, Eliana, Marqués, Luis, Bullen, Mark, y Strijbos, Jan-Willem. (2015). Let's talk about digital learners in the digital era. International Review of Research in Open and Distance Learning, 16(3), 156-187.

Gil-Salom, Luz, y Soler-Monreal, Carmen. (Eds.). (2014). Dialogicity in written specialised genres. Amsterdam, Netherland: Benjamins.

Giuse, Nunzia, Huber, Jeffrey, Guise, Dario, Brown, Clarence, Bankowtz, Richard, y Hunt, Susan. (1994). Information needs of health care professionals in an AIDS outpatient clinic as determined by chart review. Journal of the American Medical Informatics Association, 1(5), 395-403.

González, Cristina y Burdiles, Gina. (2018). Organización retórica del género Informe de Estabilidad Financiera, un contraste entre el informe del Banco Central de Chile y del Banco Federal Alemán. Círculo de Lingüistica Aplicada a la Comunicación (73), 145-160.

Graesser, Arthur, Singer, Murray, y Trabasso, Tom. (1994). Constructing inferences during narrative text comprehension. Psychological Review, 101(3), 371395.

Gunnarsson, Britt-Louise. (2009). Professional discourse. Londres, Inglaterra: Continuum.

Gunter, Barrie, Rowlands, Ian, y Nicholas, David. (2009). The Google Generation: Are ICT innovations changing information-seeking behaviour? Cambridge, Inglaterra: Chandos Publishing.

Herrera, Eugenia. (2016). Modos de hacer filosofía y modos de decir en el artículo de investigación. Un estudio comparativo de la relación entre los supuestos metodológicos y la enunciación académico-filosófica. Tesis de Magíster. Buenos Aires, Argentina: Universidad de Buenos Aires. 
Hyland, Ken. (2000). Disciplinary discourses. Social interaction in academic writing. Londres, Inglaterra: Longman.

Hyland, Ken. (2011). Academic discourse. En K. Hyland y B. Paltridge (Eds.), The continuum companion to discourse analysis (pp. 171-184). Londres, Inglaterra: Continuum.

Ito, Mizuko, Horst, Heather, Bittanti, Matteo, Boyd, Danah, Herr-Stephenson, Becky, Lange, Patricia, Pascoe, C. J., y Robinson, Laura. (2008). Living and learning with new media. Summary of findings from the digital youth project. MIT Press.

Jafre, Mohamad, Pour-Mohammadi, Majid, y Choon, Ooi. (2011). The reading habits of Malaysian Chinese university students. Journal of Studies in Education, 1(1), 1-13.

Jones, Chris, Ramanau, Ruslan, Cross, Simon, y Healing, Graham. (2010). Net generation or digital natives: Is there a distinct new generation entering university? Computers \& Education, 54(3), 722-732.

Jordan, Mark. (1981). A Preface to the Study of Philosophic Genres. Philosophy and Rhetoric, 14(4), 199-211.

Kauppinen, Antti. (2007). The rise and fall of experimental philosophy. Philosophical Explorations, 10(2), 95-118.

Kazanci, Zekeriya. (2015). University Students' Preferences of Reading from a Printed Paper or a Digital Screen - A Longitudinal Study. International Journal of Culture and History, 1, 50-53.

Kennedy, Gregor, Dalgarno, Barney, Bennett, Sue, Judd, Terry, Gray, Kathleen, y Chang, R. (2008). Immigrants and natives: Investigating differences between staff and students' use of technology. En R. Atkinson y C. McBeath (Eds.), Proceedings Hello! Where are you in the landscape of educational technology? (pp. 484-492). Melbourne, Australia: Society for Computers in Learning in Tertiary Education.

Klamer, Arjo. (1990). The textbook presentation of economic discourse. En W. Samuels (Ed.), Economics as discourse (pp. 129-154). Londres, Inglaterra: Kluwer.

Knobe, Joshua. (2004). What is experimental philosophy? The Philosophers' Magazine. 28, 37-39.

Koester, Almut. (2010). Workplace discourse. Breinigsville, PA: Continuum.

Kong, Kenneth. (2014). Professional discourse. Cambridge, Inglaterra: University Printing House.

Kwak, Duck-joo. (2010). Practising Philosophy, the Practice of Education: Exploring the Essay Form through Lukács' Soul and Form. Philosophy of Education, 44(1), 61-77.

Lavery, Jonathan. (2007). Philosophical Genres and Literary Forms: A Mildly Polemical Introduction. Poetics Today, 28(2), 171-189. 
Liceanu, Gabriel. (2017). Gatunki filozoficzne [Philosophical genres]. Przestrzenie Teorii, 27, 345-353.

Linell, Per. (1998). Discourse across boundaries: On recontextualizations and the blending of voices in professional discourse. Text. 18(2), 143-157.

López-Ferrero, Carmen, y Bach, Carmen. (2016). Discourse analysis of statements of purpose. Discourse Studies, 18, 286-310.

Machery, Edouard. (2016). What are experimental philosophers doing? Disponible en http://experimentalphilosophy.typepad.com/experimental_philosophy//2007/07/index.html.

Mangen, Anne, y Van der Weel, Adriaan. (2016). The evolution of reading in the age of digitisation: An integrative framework for reading research. Literacy, 50, 116-124.

Mangen, Anne, Walgermo, Bente, y Bronnick, Kolbjørn. (2013). Reading linear texts on paper versus computer screen: Effects on reading comprehension. International Journal of Educational Research, 58, 61-68.

McCloskey, Dreide. (1983). The rhetoric of economics. Journal of Economic Literature, 31(2) 482-504.

McCloskey, Dreide. (1994). Knowledge and persuasion in economics. Cambridge: Cambridge, Inglaterra: University Press.

Mendieta, Eduardo. (2014). Philosophy's paralipomena: Diaries, notebooks, and letters. Journal of Speculative Philosophy, 28(4), 413-421.

Ministerio de Educación, Cultura y Deporte. (2000). Estudio de hábitos de lectura y compra de libros. Madrid, España: MECD.

Ministerio de Educación, Cultura y Deporte. (2001). Estudio de hábitos de lectura y compra de libros. Madrid, España: MECD.

Molina, Marco, Vásquez-Rocca, Liliana, y Parodi, Giovanni. Relación palabragráfico en un género profesional de la economía: el caso del Informe Mensual de Estadísticas Monetarias y Financieras, Círculo de Lingüística Aplicada a la Comunicación, 76 (en prensa).

Montolío, Estrella. (2011). La comunicación escrita en la sociedad del conocimiento. Quaderns de filología. Estudis lingüistics, 16, 129-148.

Montolío, Estrella. (Dir.). (2014). Manual de escritura académica y profesional: estrategias discursivas. Barcelona, España: Ariel.

Moreno-de León, Tomás. (2017). Análisis retórico funcional del género profesional Informe Anual del Fondo Monetario Internacional. Tercer Simposio Internacional de la Cátedra UNESCO Lectura y Escritura: tensiones y desafíos en su enseñanza e investigación. Chillán, Chile.

Moyano, Estela. (Ed.) (2013). Aprender Ciencias y Humanidades: Una cuestión de lectura y escritura. Buenos Aires, Argentina: Universidad Nacional de General Sarmiento.

Naciones Unidas. (2016). Agenda 2030 y los objetivos de desarrollo sostenible. Una 
oportunidad para América Latina y el Caribe. Santiago de Chile: Publicaciones Naciones Unidas.

Nathan, Joseph, y Grossman, Sara. (2012). Professional reading habits of pharmacists attending 2 educational seminars in New York City. Journal of Pharmacy Practice, 25(6), 600-605.

Navarro, Federico. (2012). “¿Qué son los géneros profesionales? Apuntes teóricometodológicos para el estudio del discurso profesional”. En A. Cristófalo y J. Ledesma (Eds.), Actas del IV Congreso Internacional de Letras (pp. 12941303). Buenos Aires, Argentina: Universidad de Buenos Aires.

Navarro, Federico. (2015). Análisis situado del plan de negocios en español y portugués. Calidoscópio, 13(2), 189-200.

Navarro, Federico, Ávila, Natalia, Tapia-Ladino, Mónica, Cristovão, Vera, Moritz, María, Narváez Cardona, Elizabeth, y Bazerman, Charles. (2016). Panorama histórico y contrastivo de los estudios sobre lectura y escritura en educación superior publicados en América Latina. Revista Signos. Estudios de Lingüistica, 49(S1), 78-99.

Neubert, Stefan, y Reich, Kersten. (2002). Toward a constructivist theory of discourse: Rethinking the boundaries of discourse philosophy. University of Cologne. Disponible en http://konstruktivismus.uni-koeln.de/neubert_works/aufsaetze/reich_38.pdf

Nicholas, David, Rowlands, Ian, Clark, David, y Williams, Peter. (2011). Google Generation II: web behaviour experiments with the BBC. Aslib Proceedings, 63(1), 28-45.

Orna-Montesinos, Concepción. (2012). Constructing professional discourse: A multiperspective approach to domain-specific discourses. Newcastle, Inglaterra: Cambridge Scholars Publishing.

Oyarzún, Karen. (2016). Aproximación a la descripción del discurso profesional de la economía a través del Informe Económico Regional. Tesis de Licenciatura en Lengua y Literatura Hispanoamericana. Valparaíso, Chile: Pontificia Universidad Católica de Valparaíso.

Parodi, Giovanni. (2009). El Corpus Académico y Profesional del Español PUCV-2006: semejanzas y diferencias entre los géneros académicos y profesionales. Estudios Filológicos, (44), 123-147.

Parodi, Giovanni. (2010). Multisemiosis y lingüística de corpus: Artefactos (multi)semióticos en los textos de seis disciplinas en el corpus PUCV-2010. Revista de Lingüistica Teórica y Aplicada, RLA, 48(2), 33-70.

Parodi, Giovanni. (2014). Genre organization in specialized discourse: Disciplinary variation across university textbooks. Discourse Studies, 16(1), 65-87.

Parodi, Giovanni. (Ed.) (2015). Géneros académicos y géneros profesionales: Accesos discursivos para saber y hacer. Valparaíso, Chile: Ediciones Universitarias de Valparaíso. 
Parodi, Giovanni, y Burdiles, Gina. (Eds.) (2015). Leer y escribir en contextos académicos y profesionales: géneros, corpus y métodos. Santiago de Chile, Chile: Ariel.

Parodi, Giovanni, y Julio, Cristóbal. (2015). Más allá de las palabras: ¿̨Puede comprenderse el género discursivo Informe de Política Monetaria desde un único sistema semiótico predominante? Revista ALPHA, 41, 133-158.

Parodi, Giovanni, y Julio, Cristóbal. (2016). ¿Dónde se posan los ojos al leer textos multisemióticos disciplinares? Procesamiento de palabras y gráficos en un estudio experimental con eye tracker. Revista Signos. Estudios de Lingüistica, 49(S.1), 149-183.

Parodi, Giovanni, y Julio, Cristóbal. (2017). More than words: Contending semiotic systems and the role of disciplinary knowledge in specialized text comprehension. Revista Ibérica, 33, 11-36.

Parodi, Giovanni, Boudon, Enrique, y Julio, Cristóbal. (2014). La organización retórica del género manual de economía. Revista de Lingüística Teórica y Aplicada, RLA, 52(2), 133-163.

Parodi, Giovanni, Julio, Cristóbal, y Recio, Inés. (2018). When Words and Graphs Move the Eyes: The Processing of Multimodal Causal Relations. Journal of Eye Movements Research, 11(1), 1-18.

Parodi, Giovanni, Julio, Cristóbal, Nadal, Laura, Burdiles, Gina, y Cruz, Adriana. (2018). Always look back: Eye movements as a reflection of anaphoric encapsulation in Spanish while reading the neuter pronoun ello. Journal of Pragmatics, 132, 47-58.

Parodi, Giovanni, Julio, Cristóbal, y Vásquez-Rocca, Liliana. (2015). Los géneros del Corpus PUCV-UCSC-2013 del discurso académico de la economía: el caso del Informe de Política Monetaria. Revista ALED, 15(2), 179-200.

Parodi, Giovanni, Moreno-de León, Tomás, Julio, Cristóbal, y Burdiles, Gina. ¿Generación Google o generación Gutenberg?: hábitos y propósitos de lectura en estudiantes universitarios. Revista Comunicar, XVII(59) (en prensa).

Perrin, Andrew. (2016). Book reading 2016. Washington, DC, USA: Pew Research Center.

Peters, Michael. (2008). Academic Writing, Genres and Philosophy. Educational Philosophy and Theory, 40(7), 819-831.

Pieterse, Jos. (2014). Service engineers in change: Count your words. Tesis de doctorado. Netherlands: Open University of Netherlands.

Prensky, Marc. (2001). Digital natives, digital immigrants, Part 1. On the Horizon, 9(5), 1-6.

Prensky, Marc. (2009). H. Sapiens digital: From digital immigrants and digital natives to digital wisdom. Innovate: Journal of Online Education, 5(3).

Putro, Nur Hidayanto Pancoro Setyo, y Lee, Jihyun. (2017). Reading interest in a digital age, Reading Psychology, 38(8), 778-807. 
Reich, Kersten. (2000). Systemisch-konstruktivistische Pädagogik. Neuwied u.a.: Luchterhand.

Rouet, Jean-Francois, Lowe, Richard, y Schnotz, Wolfang. (Eds.). (2010). Understanding multimedia documents. Lexington, KY: Springer.

Rowlands, Ian, Nicholas, David, Williams, Peter, Huntington, Paul, Fieldhouse, Maggie, Gunter, Barrie, Withey, Richard, Jamali, Hamid, Dobrowolski, Tom, y Tenopir, Carol. (2008). The Google generation: the information behaviour of the researcher of the future. Aslib Proceedings, 60(4), 290-310.

Sahu, Hemant y Singh, Surya. (2013). Information seeking behaviour of astronomy/astrophysics scientists. Aslib Proceedings 65(2), 109-142.

Saint, Sanjay, Christakis, Dimitri, Saha, Somnath, Elmore, Joann, Welsh, Deborah, Baker, Paul, y Koepsell, Thomas. (2000). Journal reading habits of internists. Journal of General Medicine, 15(12), 881-884.

Salajan, Florin, Schönwetter, Dieter, y Cleghorn, Blaine. (2010). Student and faculty inter-generational digital divide: Fact or fiction? Computers \& Education, 55(3), 1393-1403.

Sales, Hazel. (2006). Professional communication in engineering. Basingstoke, England: Palgrave MacMillan.

Samuels, Warren. (Ed.) (1990). Economics as discourse: An analysis of the language of economists. Amsterdam, Holanda: Kluwer.

Sarangi, Srikant. (2004). Towards a communicative mentality in medical and healthcare practice. Communication and Medicine, 1(1), 1-11.

Selwyn, Neil. (2009). The digital native - myth and reality. Aslib Proceedings, 61(4), 364-379.

Shaikh, Dawn, y Chaparro, Bárbara. (2004). A survey of online reading habits of Internet users. En Proceedings of the Human Factors and Ergonomics Society Annual Meeting, 48(5), 875-879. Sage CA: Los Angeles, CA: SAGE Publications.

Silver, Marc. (2006). Language across disciplines: Towards a critical reading of contemporary academic discourse. Florida, Estados Unidos: Brown Walker Press.

Smart, Graham. (2006). Writing the economy: Activity, genre, and technology in the world of banking. Londres, Inglaterra: Equinox Publishing.

Spinuzzi, Clay. (2003). Tracing genres through organizations: a sociocultural approach to information design. Cambridge, Inglaterra: The MIT Press.

Stinson, Ray, y Mueller, Dorothy. (1980). Survey of health professionals' information habits and needs: Conducted Through Personal Interviews. JAMA The Journal of the American Medical Association, 243(2), 140-3.

Stuhr, John. (2015). Genres, Hybrids, Crossings: Mixings, Samplings, MashUps. The Journal of Speculative Philosophy, 29(1), 4-15.

Swales, John. (1990). Genre analysis. English in academic and research settings. Cambridge, Inglaterra: Cambridge University Press. 
Swales, John. (2004). Research genres: Explorations and applications. Cambridge: Cambridge University Press.

Tapia, Mónica, Ávila, Natalia, Navarro, Federico, y Bazerman, Charles. (2016). Milestones, disciplines and the future of initiatives of reading and writing in higher education: An analysis from key scholars in the field in Latin America. Ilha Do Desterro, 69(3), 189-208.

Tenopir, Carol, Volentine, Rachel, y King, Donald. (2012). Article and book reading patterns of scholars: findings for publishers. Learned Publishing, 25, 279-291.

Tenopir, Carol, King, Donald, Christian, Lisa, y Volentine, Rachel. (2015). Scholarly article seeking, reading, and use: a continuing evolution from print to electronic in the sciences and social sciences. Learned Publishing, 28(2), 93-105.

Trosborg, Anna. (Ed.) (2000). Analysing professional genres. Amsterdam, Holanda: Benjamins.

Van Hooft, Andreu. (2004). Textos y discursos de especialidad: El Español de los negocios. Amsterdam, Holanda: Rodopi.

Vásquez-Rocca, Liliana. (2016). Estudio multidimensional del género Informe de Política Monetaria en el discurso profesional de la economía. Tesis doctoral. Valparaíso, Chile: Pontificia Universidad Católica de Valparaíso.

Vásquez-Rocca, Liliana, y Parodi, Giovanni. (2015). Relaciones retóricas y multimodalidad en un género 'importado': El Informe de Política Monetaria del discurso académico de la Economía. Calidoscópio, 13(3), 388-405.

Wang, Sufang, y Bai, Xue. (2016). University students awareness, usage and attitude towards e-books: Experience from China. The Journal of Academic Librarianship, 42(3), 247-258.

Woody, William, Daniel, David, y Baker, Crystal. (2010). E-books or textbooks: Students prefer textbooks. Computers \& Education, 55, 945. 


\section{ANEXO 1.}

Resultados del análisis estadístico de proporciones para preferencia entre formato papel vs. digital de estudiantes.

\begin{tabular}{|l|c|c|c|c|c|c|c|c|c|c|}
\hline Disciplina & \multicolumn{2}{|c|}{$\begin{array}{c}\text { Preferencia } \\
\text { general }\end{array}$} & \multicolumn{2}{c|}{$\begin{array}{c}\text { Mayor } \\
\text { concentra- } \\
\text { ción }\end{array}$} & \multicolumn{2}{c|}{$\begin{array}{c}\text { Mejor } \\
\text { comprensión }\end{array}$} & \multicolumn{2}{c|}{$\begin{array}{c}\text { Mejor } \\
\text { memoriza- } \\
\text { ción }\end{array}$} & \multicolumn{2}{c|}{$\begin{array}{c}\text { Mejor } \\
\text { aprendizaje }\end{array}$} \\
\cline { 2 - 11 } & $X^{2}$ & $p$ & $X^{2}$ & $p$ & $X^{2}$ & $p$ & $X^{2}$ & $p$ & $X^{2}$ & $P$ \\
\hline $\begin{array}{l}\text { Filosofía } \\
(\mathrm{n}=44)\end{array}$ & 17.786 & $<0.0001$ & 18.711 & $<0.0001$ & 18.711 & $<0.0001$ & 22.477 & $<0.0001$ & 22.477 & $<0.0001$ \\
\hline $\begin{array}{l}\text { Economía } \\
\text { y negocios } \\
\text { (n=536) }\end{array}$ & 175.783 & $<0.0001$ & 176.528 & $<0.0001$ & 192.301 & $<0.0001$ & 197.374 & $<0.0001$ & 200.937 & $<0.0001$ \\
\hline
\end{tabular}

\section{ANEXO 2.}

Resultados del análisis estadístico de proporciones para preferencia entre formato papel vs. digital de profesionales.

\begin{tabular}{|c|c|c|c|c|}
\hline \multirow[t]{2}{*}{ Disciplina } & \multicolumn{2}{|c|}{ Lectura general } & \multicolumn{2}{|c|}{ Lectura de estudio } \\
\hline & $X^{2}$ & $P$ & $X^{2}$ & $p$ \\
\hline Filosofía (n=22) & 6.428 & 0.0112 & 9.356 & 0.0022 \\
\hline Economía y negocios $(\mathrm{n}=24)$ & 2.498 & 0.1140 & 10.906 & 0.0010 \\
\hline
\end{tabular}

